\title{
Effects of Isoproterenol and Amiodarone on the Double Potential Interval After Ablation of the Cavotricuspid Isthmus
}

\author{
HIROSHI TADA, M.D., MEHMET ÖZAYDIN, M.D., AMAN CHUGH, M.D., \\ CHRISTOPH SCHARF, M.D., HAKAN ORAL, M.D., FRANK PELOSI, JR., M.D., \\ BRADLEY P. KNIGHT, M.D., S. ADAM STRICKBERGER, M.D., and FRED MORADY, M.D. \\ From the Division of Cardiology, Department of Internal Medicine, University of Michigan, Ann Arbor, Michigan, USA
}

Double Potential Interval. Introduction: A corridor of double potentials along the ablation line has been recognized to be an indicator of complete cavotricuspid isthmus block. Isoproterenol is used to confirm cavotricuspid isthmus block, but the effects of isoproterenol on the double potential interval (DPI), either in the absence or presence of amiodarone, are unknown.

Methods and Results: Thirty-two patients with isthmus-dependent atrial flutter underwent successful ablation of the cavotricuspid isthmus. The procedure was performed in the drug-free state in 23 patients, and 2 to 7 days after discontinuation of chronic amiodarone therapy in 9 patients. Electrograms recorded along the ablation line before and during isoproterenol infusion were analyzed after isthmus block was achieved. Double potentials were recorded along the entire ablation line upon achievement of complete isthmus block in all patients. The DPI in 9 patients treated with amiodarone was longer than in the other patients $(147 \pm 32 \mathrm{msec}$ vs $119 \pm 19 \mathrm{msec}, \mathrm{P}<0.001)$. The DPI increased as the pacing cycle length shortened in patients treated with amiodarone, but not in the other patients. At all pacing cycle lengths, isoproterenol shortened the DPI to a greater extent in the patients treated with amiodarone than in the other patients.

Conclusion: Amiodarone results in rate-dependent prolongation of the DPI during coronary sinus pacing after ablation of the cavotricuspid isthmus. Isoproterenol shortens the DPI despite the presence of complete isthmus block, and this effect is accentuated in the presence of amiodarone. ( $\mathrm{J}$ Cardiovasc Electrophysiol, Vol. 14, pp. 935-939, September 2003)

atrial flutter, double potential, amiodarone, isoproterenol

Introduction

A corridor of double potentials along the ablation line is recognized to be an indicator of complete block in the cavotricuspid isthmus in patients undergoing radiofrequency catheter ablation of isthmus-dependent atrial flutter. ${ }^{1-3}$ The endpoint of complete isthmus block is more reliable when confirmed during infusion of isoproterenol. ${ }^{4}$ However, no prior studies have determined the effects of isoproterenol on double potentials along an ablation line in the cavotricuspid isthmus. Furthermore, although catheter ablation procedures usually are performed in the drug-free state, this often is not feasible in patients treated with amiodarone because of its long elimination half-life. ${ }^{5}$ The interaction between isoproterenol and amiodarone after complete block in the cavotricuspid isthmus also has not been determined. Therefore, the aim of this study was to examine the effects of isoproterenol on the double potential interval after the creation of cavotricuspid isthmus block, both in the absence and presence of amiodarone.

Supported by a grant from the Gunma Prefecture Government and by the Ellen and Robert Thompson Atrial Fibrillation Research Fund.

Address for correspondence: Fred Morady, M.D., Division of Cardiology, Department of Internal Medicine, University of Michigan, B1F245, 1500 E. Medical Center Drive, Ann Arbor, MI 48109-0022. Fax: 734-936-7026. E-mail:fmorady@umich.edu

Manuscript received 13 June 2002; Accepted for publication 24 June 2003.

\section{Methods}

\section{Characteristics of Subjects}

The subjects of this study were 32 patients ( 23 men and 9 women; mean age $59 \pm 14$ years $[ \pm \mathrm{SD}]$ ) who underwent radiofrequency catheter ablation of isthmus-dependent atrial flutter at the University of Michigan Medical Center. Structural heart disease was present in 9 patients (28\%): coronary artery disease in 4 , nonischemic cardiomyopathy in 2 , and hypertensive heart disease in 3 . Treatment with all antiarrhythmic medications was discontinued for at least five half-lives in 23 patients who had not been treated with amiodarone. In 9 patients who had been treated with amiodarone for at least 6 months, amiodarone was withheld for 2 to 7 days before the ablation procedure. The dosage of amiodarone was $200 \mathrm{mg} /$ day in all of the patients who had been treated with amiodarone.

\section{Catheter Ablation Procedure}

All patients provided informed consent before the electrophysiologic procedure. A 7-French duodecapolar halo catheter (2-mm spacing between electrodes of each bipole; spacing between pairs of electrodes $=20-2-2-2-2-2-2-25-25-$ $25 \mathrm{~mm}$, Daig Corporation, Minnetonka, MN, USA) was positioned along the tricuspid annulus with the distal bipole of the halo catheter within the proximal portion of the coronary sinus. A 7-French quadripolar catheter (EP Technologies, San Jose, CA, USA) was positioned within the proximal coronary sinus for pacing. A 7-French quadripolar catheter with a 
deflectable tip, 4-mm distal electrode (2-5-2 $\mathrm{mm}$ interelectrode spacing), and a thermistor embedded in the distal electrode (EP Technologies) was used for mapping the isthmus and delivering radiofrequency energy.

Bipolar electrograms were filtered at settings of 30 to $500 \mathrm{~Hz}$ and recorded digitally (EPMed Systems, Mount Arlington, NJ, USA). Electrograms were analyzed offline at a gain setting of 0.1 to $0.2 \mathrm{mV} / \mathrm{cm}$ and at a sweep speed of 100 to $200 \mathrm{~mm} / \mathrm{sec}$. Coronary sinus pacing was performed with a stimulator (model EP-3 clinical stimulator, EPMed Systems) at twice diastolic threshold and with a pulse width of $2 \mathrm{msec}$.

Under fluoroscopic guidance, contiguous applications of radiofrequency energy were delivered to create a line of block in the cavotricuspid isthmus. The power was automatically adjusted to maintain a target temperature of $60^{\circ} \mathrm{C}$ at the electrode-tissue interface. Each application of energy was 45 to 60 seconds in duration.

The endpoint of the procedure was complete isthmus block in the clockwise and counterclockwise directions. This endpoint was attained in all patients in this study. The criteria for complete block were assessed during coronary sinus pacing at a cycle length of $600 \mathrm{msec}$ and consisted of an appropriate atrial activation sequence recorded with the halo catheter, ${ }^{6}$ negative initial polarity of the bipolar electrograms recorded just lateral to the ablation line during coronary sinus pacing, ${ }^{7}$ and double potentials with a double potential interval $>110$ msec along the entire ablation line, or with an isoelectric double potential interval $>90$ msec. $^{3}$

Because of a history of atrial fibrillation, 3 patients were treated with amiodarone and 3 patients were treated with propafenone after flutter ablation. During a mean follow-up of $13 \pm 6$ months, none of the patients in this study had a recurrence of atrial flutter.

\section{Study Protocol}

After complete cavotricuspid isthmus block was achieved, the ablation catheter was positioned at a site along the ablation line at which a double potential was readily seen. Coronary sinus pacing was performed at cycle lengths of 500, 400, and $350 \mathrm{msec}$ before and during infusion of 2 to $8 \mu \mathrm{g} / \mathrm{min}$ of isoproterenol, titrated to shorten the sinus cycle length by $30 \%$ to $35 \%$.

The double potential interval was defined as the interval separating the two components of a double potential (Fig. 1). The double potential interval was measured with electronic calipers from the peak of the first component of the double potential to the peak of the second component, as previously described. ${ }^{3}$ Three double potential intervals were measured and averaged for each measurement.

\section{Statistical Analysis}

Continuous variables are expressed as mean \pm 1 SD. Double potential intervals were compared using Student's $t$-test, paired $t$-test, or analysis of variance with repeated measures, as appropriate. $\mathrm{P}<0.05$ was considered statistically significant.

\section{Results}

\section{Double Potential Intervals in the Absence of Amiodarone}

Mean double potential interval at a pacing cycle length of $500 \mathrm{msec}$ in the patients not treated with amiodarone was
Before Iso

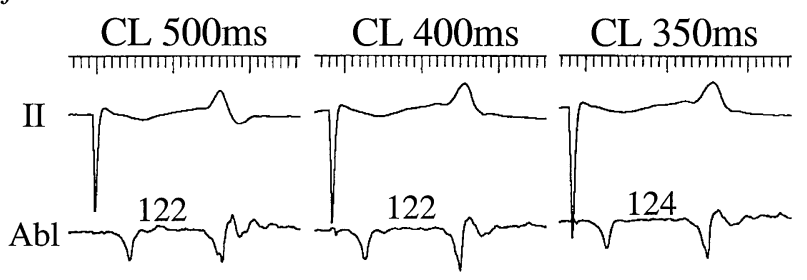

During Iso

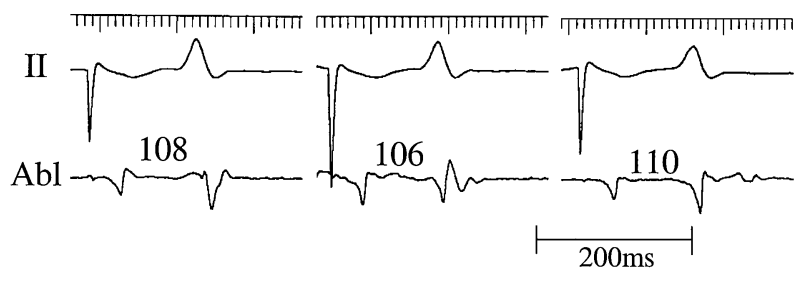

Figure 1. Double potential interval recorded along an ablation line in the cavotricuspid isthmus during coronary sinus pacing at cycle lengths $(C L)$ of 500, 400, and $350 \mathrm{msec}$, before and during infusion of isoproterenol (Iso). Shown are leads II and a recording obtained with the ablation catheter (Abl) positioned on the ablation line in the cavotricuspid isthmus. This patient had not been treated with amiodarone.

$119 \pm 19$ msec. Double potential interval did not change significantly during pacing at cycle lengths of 400 and 350 msec (Figs. 1 and 2).

During infusion of isoproterenol, mean sinus cycle length shortened from $908 \pm 157 \mathrm{msec}$ to $633 \pm 94 \mathrm{msec}(\mathrm{P}<$ 0.001 ). Mean double potential interval at a pacing cycle length of $500 \mathrm{msec}$ during isoproterenol infusion shortened from $119 \pm 19 \mathrm{msec}$ to $111 \pm 17 \mathrm{msec}(\mathrm{P}<0.01$, Figs. 1 and 2). Mean double potential interval at pacing cycle lengths of 400 and $350 \mathrm{msec}$ also were significantly shorter during than before infusion of isoproterenol $(\mathrm{P}<0.01$, Fig. 2).

\section{Double Potential Intervals in the Presence of Amiodarone}

Mean double potential interval at a pacing cycle length of $500 \mathrm{msec}$ in the patients treated with amiodarone was

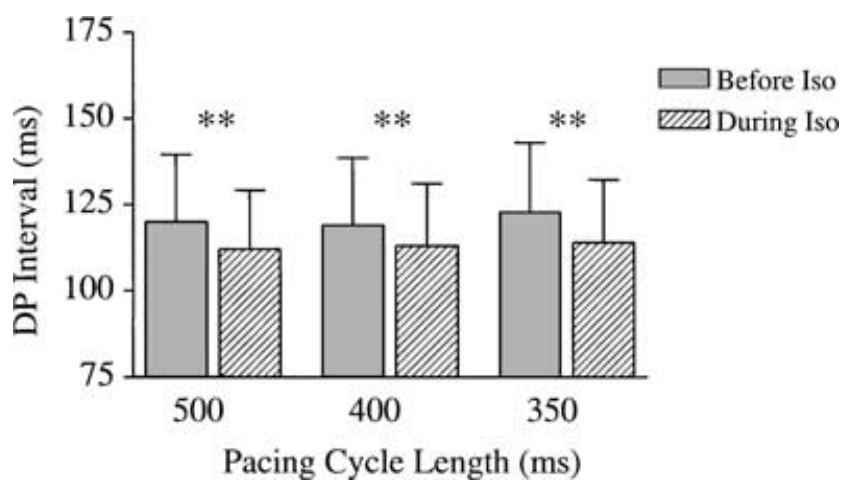

Figure 2. Double potential (DP) intervals at pacing cycle lengths of 500, 400, and 350 msec before and during infusion of isoproterenol (Iso), among 31 patients not treated with amiodarone. Error bars represent $1 S D .{ }^{* *} P<$ 0.01 (before Iso vs during Iso). 


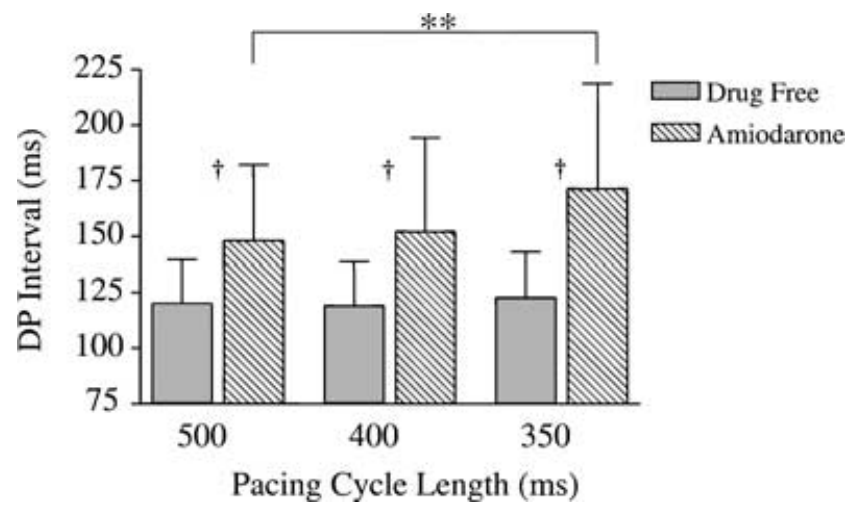

Figure 3. Double potential (DP) intervals at pacing cycle lengths of 500, 400, and 350 msec in 31 patients who were in the drug-free state and in 12 patients who were treated with amiodarone. Error bars represent $1 S D$. ${ }^{* *} P$ $<0.01 ; \dagger P<0.001$.

$147 \pm 32 \mathrm{msec}$, which was significantly longer than the corresponding double potential interval in the patients not treated with amiodarone ( $\mathrm{P}<0.001$, Fig. 3). Double potential interval lengthened significantly as the pacing cycle length shortened to $350 \mathrm{msec}$, and double potential intervals at pacing cycle lengths of 400 and $350 \mathrm{msec}$ also were significantly longer than the corresponding double potential intervals in the patients who were in the drug-free state $(\mathrm{P}<0.001$, Figs. 3 and 4).

During infusion of isoproterenol, mean sinus cycle length shortened from $1050 \pm 221 \mathrm{msec}$ to $681 \pm 77 \mathrm{msec}$ in the patients treated with amiodarone $(\mathrm{P}<0.001)$. Among the patients treated with amiodarone, mean double potential interval at a pacing cycle length of $500 \mathrm{msec}$ shortened significantly during infusion of isoproterenol to $134 \pm 26 \mathrm{msec}$ $(\mathrm{P}<0.05$, Figs. 4 and 5). Mean double potential interval at pacing cycle lengths of 400 and $350 \mathrm{msec}$ also was significantly shorter during than before infusion of isoproterenol $(\mathrm{P}<0.01$, Fig. 5).

\section{Before Iso}
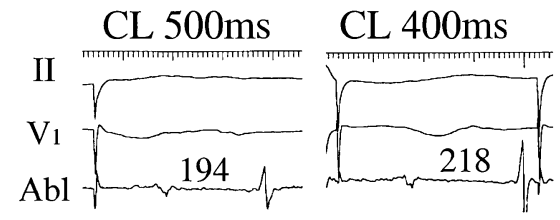

\section{350ms}

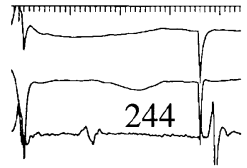

During Iso
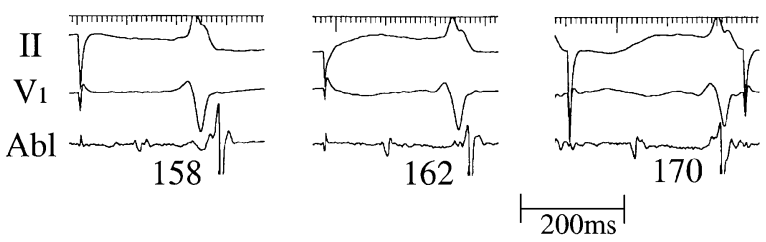

Figure 4. Double potential interval recorded along an ablation line in the cavotricuspid isthmus during coronary sinus pacing at cycle lengths (CL) of 500, 400, and $350 \mathrm{msec}$, before and during infusion of isoproterenol (Iso). This patient was treated with amiodarone. Shown are leads II and $V_{I}$ and a recording obtained with the ablation catheter $(A b l)$ positioned on the ablation line in the cavotricuspid isthmus.

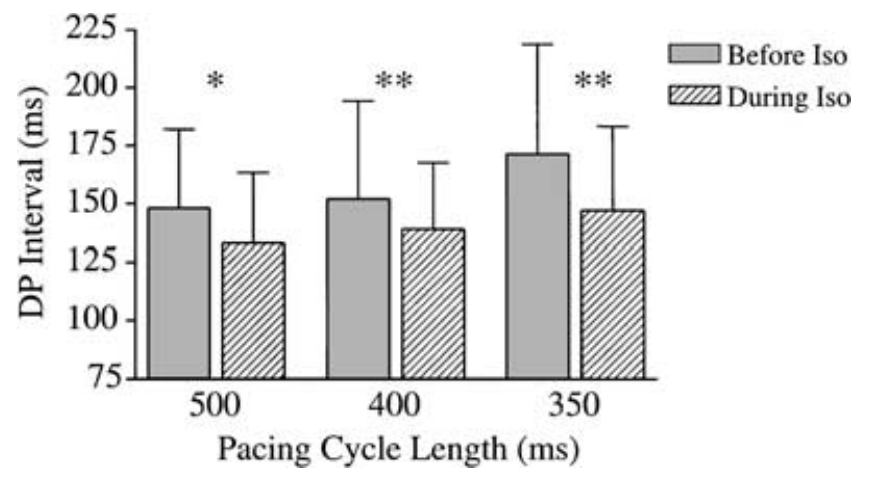

Figure 5. Double potential (DP) intervals at pacing cycle lengths of 500, 400, and 350 msec before and during infusion of isoproterenol (Iso), among 12 patients treated with amiodarone. Error bars represent $1 S D .{ }^{*} P<0.05$; ${ }^{* *} P<0.01$.

\section{Discussion}

\section{Main Findings}

The results of this study demonstrate that amiodarone lengthens the double potential interval that is recorded in the cavotricuspid isthmus after complete isthmus block is achieved by radiofrequency catheter ablation. Double potential intervals were $19 \%$ to $29 \%$ longer among patients treated with amiodarone, depending on the pacing cycle length. Furthermore, whereas isoproterenol shortened the double potential interval by only $5 \%$ to $7 \%$ in patients who were in the drug-free state, it had a more pronounced effect in patients who were treated with amiodarone. Depending on the pacing cycle length, the double potential interval shortened by $9 \%$ to $12 \%$ during infusion of isoproterenol among patients treated with amiodarone.

\section{Double Potential Intervals as an Indicator of Complete Isthmus Block}

A prior study demonstrated that a parallel corridor of double potentials separated by at least $110 \mathrm{msec}$ or by an isoelectric interval of at least $90 \mathrm{msec}$ along an ablation line during coronary sinus pacing is a reliable indicator of complete block in the cavotricuspid isthmus. ${ }^{3}$ However, in that study, all patients were in the drug-free state. Amiodarone is often used to treat atrial flutter, and because of its prolonged elimination half-life, ${ }^{5}$ it is not practical to wait for complete drug washout before performing radiofrequency catheter ablation in patients who have been drug refractory. The results of this study demonstrate that amiodarone significantly lengthens the double potential interval measured in the cavotricuspid isthmus during coronary sinus pacing.

Of note is that the data in this study were obtained only after complete isthmus block had been achieved, and complete block was confirmed by an appropriate atrial activation sequence and by changes in electrogram polarity during coronary sinus pacing. Because the double potential interval after block was achieved was lengthened by amiodarone, it is likely that amiodarone also lengthened the double potential interval when isthmus block was still incomplete. Therefore, the findings of this study imply that the double potential interval criteria of $\beta \leq 90$ to $110 \mathrm{msec}$ 
that are indicative of complete isthmus block are not applicable to patients who have recently been treated with amiodarone.

\section{Response to Isoproterenol}

Isoproterenol has been used to detect incomplete isthmus block in patients undergoing catheter ablation of the cavotricuspid isthmus. ${ }^{4}$ Among patients in this study who were in the drug-free state, isoproterenol had a minimal effect on the double potential intervals along the ablation line after complete isthmus block had been achieved. Before infusion of isoproterenol, the minimum isoelectric double potential interval during coronary sinus pacing at a cycle length of $500 \mathrm{msec}$ was $90 \mathrm{msec}$, and the minimum nonisoelectric double potential interval was $110 \mathrm{msec}$. When isoproterenol was infused, the minimum isopotential double potential interval shortened to $88 \mathrm{msec}$, and the minimum nonisoelectric double potential interval shortened to $104 \mathrm{msec}$. Therefore, the double potential interval criteria for complete isthmus block should be shortened by approximately $5 \mathrm{msec}$ when isoproterenol is infused.

The effect of isoproterenol on the double potential interval was augmented among the patients in this study who were treated with amiodarone. Despite shortening of the double potential interval by as much as $75 \mathrm{msec}$, all patients still had evidence of complete isthmus block based on criteria that were independent of the double potential interval. Therefore, a marked shortening of the double potential interval does not necessarily indicate incomplete isthmus block among patients who have been treated with amiodarone.

Sympathetic activation by isoproterenol or epinephrine has been demonstrated to reverse the pharmacologic effects of several antiarrhythmic drugs, including verapamil, quinidine, procainamide, and flecainide. ${ }^{8-13}$ Because amiodarone is a $\beta$-adrenergic antagonist, ${ }^{14}$ its electrophysiologic effects in the ventricle have been found to be more resistant to sympathetic activation than the effects of other drugs. ${ }^{15,16}$ Nevertheless, amiodarone's effects on ventricular conduction and refractoriness are partially attenuated by isoproterenol or epinephrine..$^{15,16}$ The results of the present study demonstrate that amiodarone's effect on atrial conduction also is partially reversed by isoproterenol.

\section{Decremental Conduction in the Isthmus}

A prior study demonstrated that conduction across the cavotricuspid isthmus is not decremental. ${ }^{17}$ In the present study, in drug-free patients, the double potential interval after complete cavotricuspid isthmus block was achieved was found to be stable during incremental coronary sinus pacing, consistent with an absence of decremental conduction in the cavotricuspid isthmus and in the atrial tissue surrounding the tricuspid annulus. However, the lengthening of the double potential interval during incremental coronary sinus pacing in patients treated with amiodarone indicates that this drug induced decremental conduction in the cavotricuspid isthmus and/or other areas in the right atrium. This effect of amiodarone is attributable to frequency-dependent blockade of sodium channels. ${ }^{18}$

\section{Study Limitations}

The effect of amiodarone on the double potential interval was assessed only after complete isthmus block was achieved. Therefore, this study does not provide information on the value of the double potential interval for discriminating incomplete from complete isthmus block in patients recently treated with amiodarone.

\section{Conclusion}

When isoproterenol is used to confirm complete block in the cavotricuspid isthmus, the double potential interval criteria of 90 to $110 \mathrm{msec}$ should be adjusted downward by approximately $5 \mathrm{msec}$. However, if the patient has been recently treated with amiodarone, the double potential interval during isoproterenol infusion may shorten by as much as $75 \mathrm{msec}$ despite the presence of complete cavotricuspid isthmus block. Therefore, when atrial flutter ablation is performed in the presence of amiodarone, criteria for isthmus block that are not influenced by isoproterenol, namely, the atrial activation sequence ${ }^{6}$ and electrogram polarity, ${ }^{7}$ may be more reliable than the double potential interval.

\section{References}

1. Shah DC, Haissaguerre M, Jais P, Fischer B, Takahashi A, Hocini M, Clementy J: Simplified electrophysiologically directed catheter ablation of recurrent common atrial flutter. Circulation 1997;96:2505-2508.

2. Shah DC, Takahashi A, Jais P, Hocini M, Clementy J, Haissaguerre M: Local electrogram-based criteria of cavotricuspid isthmus block. J Cardiovasc Electrophysiol 1999;10:662-669.

3. Tada H, Oral H, Sticherling C, Chough SP, Baker RL, Wasmer K, Pelosi F Jr, Knight BP, Strickberger SA, Morady F: Double potentials along the ablation line as a guide to radiofrequency ablation of typical atrial flutter. J Am Coll Cardiol 2001;38:750-755.

4. Nabar A, Rodriguez LM, Timmermans C, Smeets JL, Wellens HJ: Isoproterenol to evaluate resumption of conduction after right atrial isthmus ablation in type I atrial flutter. Circulation 1999;99:3286-3291.

5. Pollak PT, Bouillon T, Shafer SL: Population pharmacokinetics of long-term oral amiodarone therapy. Clin Pharmacol Ther 2000;67:642652.

6. Chen J, de Chillou C, Basiouny T, Sadoul N, Filho JD, Magnin-Poull I, Messier M, Aliot E: Cavotricuspid isthmus mapping to assess bidirectional block during common atrial flutter radiofrequency ablation. Circulation 1999;100:2507-2513.

7. Tada H, Oral H, Sticherling C, Chough SP, Baker RL, Wasmer K, Kim MH, Pelosi F Jr, Michaud GF, Knight BP, Strickberger SA, Morady F: Electrogram polarity and cavotricuspid isthmus block during ablation of typical atrial flutter. J Cardiovasc Electrophysiol 2001;12:393399.

8. Morady F, Kou WH, Kadish AH, Toivonen LK, Kushner JA, Schmaltz $\mathrm{S}$ : Effects of epinephrine in patients with an accessory atrioventricular connection treated with quinidine. Am J Cardiol 1988;62:580584.

9. Morady F, Kou WH, Kadish AH, Nelson SD, Toivonen LK, Kushner JA, Schmaltz S, de Buitleir M: Antagonism of quinidine's electrophysiologic effects by epinephrine in patients with ventricular tachycardia. J Am Coll Cardiol 1988;12:388-394.

10. Jazayeri MR, Van Wyhe G, Avitall B, McKinnie J, Tchou P, Akhtar M: Isoproterenol reversal of antiarrhythmic effects in patients with inducible sustained ventricular tachyarrhythmias. J Am Coll Cardiol 1989;14:705-711.

11. Manolis AS, Estes NA 3rd: Reversal of electrophysiologic effects of flecainide on the accessory pathway by isoproterenol in the WolffParkinson-White syndrome. Am J Cardiol 1989;64:194-198.

12. Morady F, Kou WH, Kadish AH, Toivonen LK, Kushner JA, Schmaltz S: Epinephrine-induced reversal of verapamil's electrophysiologic and therapeutic effects in patients with paroxysmal supraventricular tachycardia. Circulation 1989;79:783-790. 
13. Markel ML, Miles WM, Luck JC, Klein LS, Prystowsky EN: Differential effects of isoproterenol on sustained ventricular tachycardia before and during procainamide and quinidine antiarrhythmic drug therapy. Circulation 1993;87:783-792.

14. Kadish AH, Chen RF, Schmaltz S, Morady F: Magnitude and time course of beta-adrenergic antagonism during oral amiodarone therapy. J Am Coll Cardiol 1990;16:1240-1245.

15. Calkins H, Sousa J, el-Atassi R, Schmaltz S, Kadish A, Morady F: Reversal of antiarrhythmic drug effects by epinephrine: quinidine versus amiodarone. J Am Coll Cardiol 1992;19:347352.
16. Sager PT, Follmer C, Uppal P, Pruitt C, Godfrey R: The effects of beta-adrenergic stimulation on the frequency-dependent electrophysiologic actions of amiodarone and sematilide in humans. Circulation 1994;90:1811-1819.

17. Kinder C, Kall J, Kopp D, Rubenstein D, Burke M, Wilber D: Conduction properties of the inferior vena cava-tricuspid annular isthmus in patients with typical atrial flutter. J Cardiovasc Electrophysiol 1997;8:727737.

18. Sager PT, Uppal P, Follmer C, Antimisiaris M, Pruitt C, Singh BN: The frequency-dependent electrophysiologic effects of amiodarone in humans. Circulation 1993;88:1063-1071. 